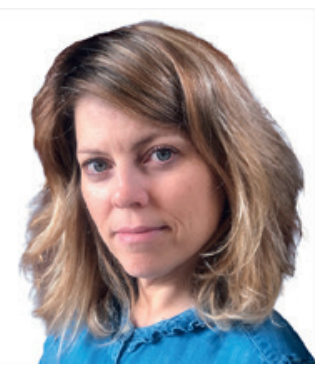

\title{
Malnutrition in Inflammatory Bowel Diseases. What do we know today?
}

Christina N. Katsagoni

Clinical Dietitian at Agia Sofia Children's Hospital, Athens, Greece

Abstract from Wong K, Isaac DM, Wine E: Growth Delay in Inflammatory Bowel Diseases: Significance, Causes, and Management. Dig Dis Sci 2021;66(4):954-964.

\section{Keywords}

Bone mass · Crohn disease · Height · Puberty - Ulcerative colitis . Weight

\section{Abstract}

Growth delay with height and weight impairment is a common feature of pediatric inflammatory bowel diseases (PIBD). Up to 2/3 of Crohn Disease patients have impaired weight at diagnosis, and up to $1 / 3$ have impaired height. Ulcerative colitis usually manifests earlier with less impaired growth, though patients can be affected. Ultimately, growth delay, if not corrected, can reduce final adult height. Weight loss, reduced bone mass, and pubertal delay are also concerns associated with growth delay in newly diagnosed PIBD patients. The mechanisms for growth delay in IBD are multifactorial and include reduced nutrient intake, poor absorption, increased fecal losses, as well as direct effects from inflam-
\end{abstract}

mation and treatment modalities. Management of growth delay requires optimal disease control. Exclusive enteral nutrition (EEN), biologic therapy, and corticosteroids are the primary induction strategies used in PIBD, and both EEN and biologics positively impact growth and bone development. Beyond adequate disease control, growth delay and pubertal delay require a multidisciplinary approach, dependent on diligent monitoring and identification, nutritional rehabilitation, and involvement of endocrinology and psychiatry services as needed. Pitfalls that clinicians may encounter when managing growth delay include refeeding syndrome, obesity (even in the setting of malnutrition), and restrictive diets. Although treatment of PIBD has improved substantially in the last several decades with the era of biologic therapies and EEN, there is still much to be learned about growth delay in $\mathrm{PIBD}$ in order to improve outcomes.

(c) 2020 The Author(s) 


\section{Knowledge Transfer}

\section{Background}

Inflammatory bowel diseases (IBD) are chronic, often relapsing, conditions. Paediatric IBD (PIBD) is associated with more severe morbidity and a more complicated disease process than adult-onset IBD [1]. The clinical presentation of PIBD varies, including both gastrointestinal (e.g. bloody diarrhoea/abdominal pain in ulcerative colitis (UC) patients or, depending on disease location, even no specific symptoms in Crohn's disease (CD) patients) and extraintestinal (e.g. oral aphthous ulcerations, dermatologic conditions, arthritis) symptoms. Consequently, children with PIBD are at risk of malnutrition, including macronutrient and micronutrient deficiencies, leading to poor growth and often delayed puberty.

\section{Review Results}

In the review by Wong and colleagues published in the Digestive Diseases and Sciences Journal [2], the authors describe the clinical presentation of growth delay in PIBD as well as its possible mechanisms and management options (summarized in Fig. 1). The mentioned pitfalls which may occur while treating malnutrition in PIBD are especially interesting for clinical practice.

\section{Clinical Presentation of Growth Delay in PIBD}

Weight loss leading to growth failure is observed more often in CD patients than in UC patients, the reason lying in the very nature of the diseases. Every month of delay, from disease onset to diagnosis, is associated with a decrease in height for age. Nevertheless, if remission is achieved, it seems that there is a "therapeutic window" related to growth, i.e. children can continue to grow beyond the chronologic age at which epiphyseal closure is defined to occur. However, metabolic bone disease is often seen in PIBD, which is associated with decreased bone mineral density (BMD). Consequently, PIBD is associated with suboptimal linear growth which is more evident in CD patients, and may lead to delayed puberty.

\section{Pathogenesis of Growth Delay}

Growth delay or delayed puberty in PIBD is multifactorial: Inflammation is a well-documented factor as several pro-inflammatory cytokines affect growth-related hormones, reduce appetite, and increase the metabolic activity of macronutrients. Malabsorption and maldigestion are risk factors for growth failure as well, particularly in the case of small bowel disease location, leading to nutrient and protein losses. Symptoms such as pain, nausea, or diarrhoea may reduce patients' food intake and influence their food preferences. Increased resting energy expenditure is thought to further contribute to increased calorie expenditure; however, this point remains controversial. Other factors such as side effects from medication, alterations in the gut microbiome, as well as physiological factors may also impact on the growth of PIBD patients.

\section{Management of Growth Delay}

Exclusive enteral nutrition (EEN) is the first-line therapy for inducing clinical remission in CD. It is given solely as a nutritionally complete formula for 6-8 weeks. While comparable solid food diets for CD patients are promising, EEN is considered equal or even superior to corticosteroid induction therapy. Still, corticosteroids remain an important therapeutic choice. Anti-TNF agents are used as induction

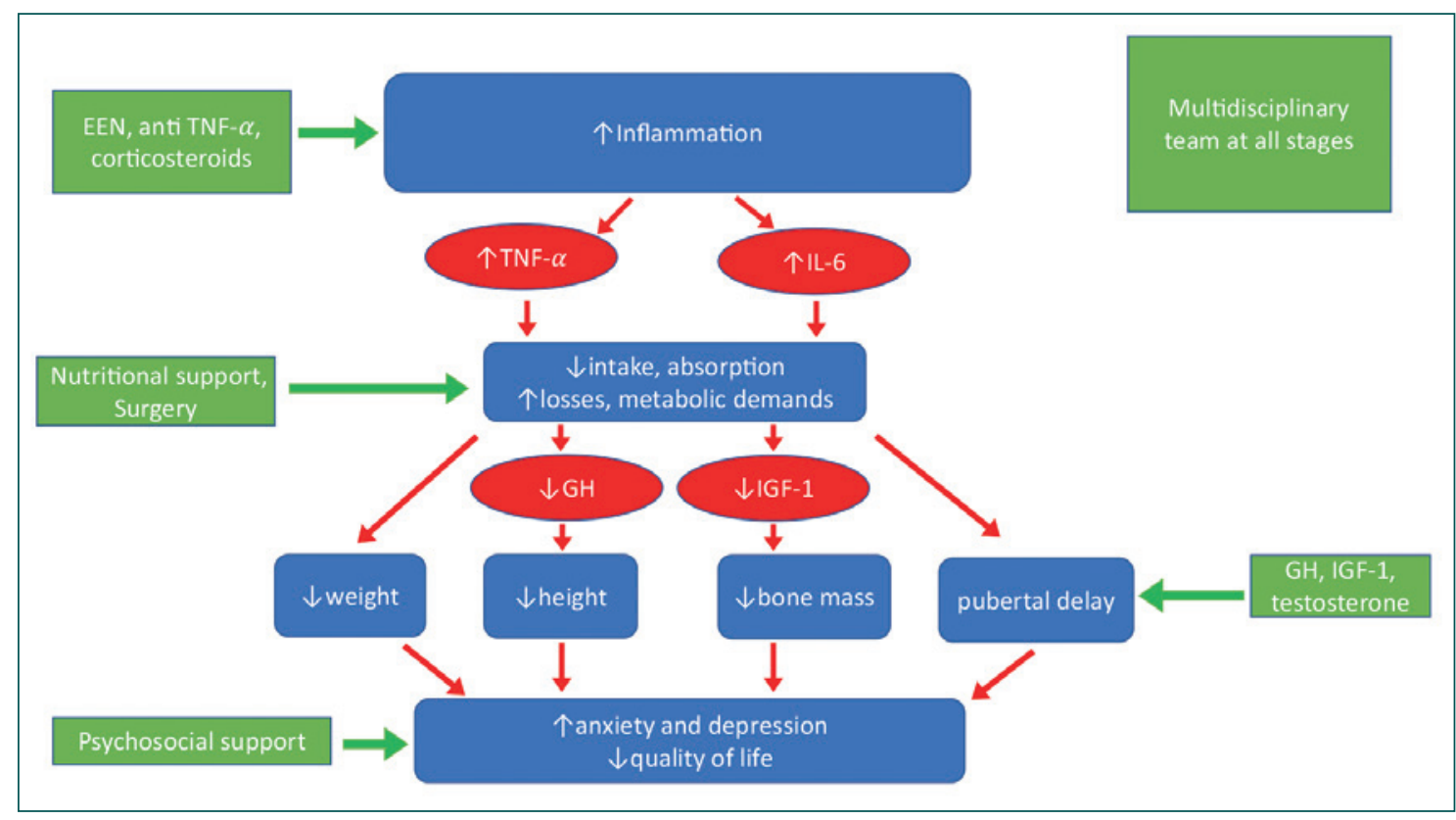

Fig. 1. Overview of clinical manifestations, mechanisms, and interventions in PIBD growth delay: Schematic representation of factors that impact growth delay and its consequences. Not all pathways are completely established; details are provided in the text. Blue: clinical manifestations, Red: mechanisms and biomarkers, Green: interventions [2]. 
therapy in severe PIBD and have been associated with improved growth. In cases of severe disease refractory to aggressive medical treatment, surgery has been correlated with improvements in linear growth. Overall, PIBD patients should follow the same age-specific dietary recommendations as healthy children, whereas during flares an increase in protein intake by $25 \%$ is recommended.

\section{Management of Pubertal Delay}

There are reports on endocrine therapies including sex hormones, growth hormones, and insulin-like growth factor-1 for the treatment of growth failure and subsequent pubertal delay in PIBD patients. However, more research is needed before these therapies can be included in clinical practice.

\section{Pitfalls in Managing Weight and Growth Problems}

Refeeding syndrome can occur after rapid refeeding in some PIBD patients, especially those with severe weight loss. This leads to severe hypophosphatemia and metabolic abnormalities, and therefore requires particular attention and caution.

On the other hand, obesity is also increasingly seen in IBD patients. Uncontrolled eating after diagnosis, misconceptions of what someone with IBD can or cannot eat, and adherence to a restricted diet without professional supervision in order to induce or maintain remission can lead to insufficient energy and macro-/micronutrient intake.

In conclusion, due to the dynamic and complex course of IBD, the treatment of paediatric patients with IBD should follow a multidisciplinary approach including paediatric gastroenterologists, nurses, registered dietitians, social workers, and psychologists.

\section{Disclosure Statement}

I hereby declare that there are no conflicts of interest with regard to this commentary.

\section{References}

1 Oliveira SB, Monteiro IM. Diagnosis and management of inflammatory bowel disease in children. BMJ. 2017 May 31;357:j2083.

2 Wong K, Isaac DM, Wine E. Growth Delay in Inflammatory Bowel Diseases: Significance, Causes, and Management. Dig Dis Sci. 2021;66(4):954-964.

Correspondence to:

Dr. Christina N. Katsagoni, christina.katsagoni@gmail.com 